\begin{tabular}{|c|c|}
\hline Title & $\begin{array}{l}\text { The effect of ovariectomy on urethral continence mechanisms during sneeze reflex in middle aged versus young adult } \\
\text { rats }\end{array}$ \\
\hline Author(s) & Kitta, Takeya; Y oshikawa, Satoru; Kawamorita, Naoki; de Groat, William C.; Nonomura, Katsuya; Y oshimura, Naoki \\
\hline Citation & $\begin{array}{l}\text { Neurourology and Urodynamics, 35(1), 122-127 } \\
\text { https://doi.org/10.1002/hau.22690 }\end{array}$ \\
\hline Issue Date & 2016-01 \\
\hline Doc URL & http:/hdl. handle.net/2115/63973 \\
\hline Rights & $\begin{array}{l}\text { This is the peer reviewed version of the following article: Neurourology and Urodynamics, } V \text { olume } 35 \text {, Issue } 1 \text {, pages } \\
122-127,2016 \text {, which has been published in final form at http://dx.doi.org/10.1002/hau.22690. This article may be used } \\
\text { for non-commercial purposes in accordance with Wiley Terms and Conditions for Self-A rchiving. }\end{array}$ \\
\hline Type & article (author version) \\
\hline File Information & NeurourolUrodyn35_122.pdf \\
\hline
\end{tabular}

Instructions for use 


\section{The effect of ovariectomy on urethral continence mechanisms during sneeze reflex in}

\section{middle-aged versus young adult rats}

Takeya Kitta ${ }^{1,2}$, Satoru Yoshikawa ${ }^{1}$, Naoki Kawamorita ${ }^{1}$, William C. de Groat ${ }^{3}$, Katsuya Nonomura ${ }^{2}$, Naoki Yoshimura ${ }^{1,3}$

1. Department of Urology, University of Pittsburgh, Pittsburgh, PA, USA 15213

2. Department of Renal and Genitourinary Surgery, Graduate School of Medicine, Hokkaido

University, Sapporo, Japan 060-8638

3. Department of Pharmacology and Chemical Biology, University of Pittsburgh, Pittsburgh, PA, USA 15260

Corresponding author: Naoki Yoshimura, MD, PhD.

Department of Urology University of Pittsburgh School of Medicine

Suite 700, Kaufmann Medical Bldg.

3471 Fifth Ave, Pittsburgh, PA 15213

Phone: 412-692-4137 Fax: 412-692-4380

E-mail: nyos@pitt.edu 
Running Head: Continence mechanisms in middle-aged and young adult rats 


\begin{abstract}
Aims Stress urinary incontinence (SUI) is common in post-menopausal women. The present study therefore examined how aging and estrogen deficiency induced by ovariectomy (OVX) affect the urethral continence mechanism that prevents sneeze-induced SUI in rats.

Methods Young (3 months old) and middle-aged (12 months old) female rats underwent bilateral OVX or sham operation. Urethral activity was measured by the amplitude of urethral responses during sneezing (A-URS) and urethral baseline pressure (UBP). Apoptotic changes in urethral tissue sections were examined by the TUNEL method.

Results In middle-aged rats, UBP, but not A-URS, was significantly decreased compared to young rats. In 3-week OVX rats, A-URS was significantly decreased compared to sham rats in both young and middle-aged groups, and the OVX-induced reduction in A-URS was more pronounced in middle-aged rats. Neither young 3-week OVX nor sham rats leaked during sneezing; however, SUI occurred in 2/8 middle-aged rats with 3-week OVX, and after 6 weeks of OVX, SUI was observed in 5/8 young rats and 6/8 middle-aged rats. In middle-aged rats, TUNEL positive cells were significantly increased in urethral striated muscles whereas, after OVX, the increased number of positive cells was also found in the mucosa.
\end{abstract}

Conclusions These results indicate that aging is more likely to impair baseline urethral function than striated muscle-mediated reflex activity although apoptotic changes are found in urethral striated muscle. Estrogen deficiency additionally impairs the striated muscle-mediated continence reflex. Thus, aging and estrogen deficiency differently and 
additively affect baseline urethral function and neurally-evoked, striated muscle-mediated urethral continence mechanisms to induce SUI. 
Keywords: Stress urinary incontinence, ovariectomy, sneeze, rat, apoptosis 


\section{Introduction}

Stress urinary incontinence (SUI) is common in post-menopausal women, suggesting that aging and estrogen deficiency contribute to its etiology $(1,2)$. Circulating estrogen levels fall at menopause, but there is conflicting evidence whether voiding symptoms in these women are related to hypoestrogenism or to aging per se. Moreover, limited information is available for detailed pathophysiological effects of these etiological factors on urethral function.

Our group previously reported in rats that an active urethral closure mechanism prevents SUI during the sneeze reflex, and that the sneeze-induced continence reflex is mediated by somatic nerve-induced reflex contractions of the external urethral sphincter and pelvic floor striated muscles (3). Using this model system, we also reported that ovariectomy (OVX) impairs the urethral function by reducing urethral baseline pressure (UBP) and urethral responses to sneezing to induce sneeze-induced SUI at 6 weeks after OVX in young rats (4). However, it is not known whether OVX-induced changes in the urethral continence function are affected by the aging process.

The present study therefore utilized physiological and histological methods to examine how aging and estrogen deficiency induced by OVX affect the urethral continence mechanism that prevents sneeze-induced SUI using young and middle-aged rats. 


\section{Materials and methods}

\section{Animals, study design}

Young (3 months old) and middle-aged (12 months old) female Sprague-Dawley rats underwent bilateral OVX or sham operation (laparotomy and wound closure) as previously reported (4). After 3 or 6 weeks of OVX, urethral responses and immunohistochemical analyses were performed. The experimental schedule and the number of animals are shown in Fig. 1. Although we used sham operated rats as a control group in young rats, intact rats were used as controls in the middle-aged group because our preliminary experiments in middle-aged rats did not reveal significant differences in functional and morphological properties of the urethra between sham operated and intact rats. All experimental protocols were approved by the University of Pittsburgh Institutional Animal Care and Use Committee.

\section{Surgical Procedures for experiments}

The surgical techniques for urethral function testing were similar to those described

previously $(4,5)$. Briefly, under isoflurane anesthesia, a polyethylene catheter (PE-10, Clay-Adams, Parsippany, NJ) was inserted into the jugular vein for maintaining anesthesia. The bladder was exposed through an abdominal incision, ureters were cut bilaterally, and their distal ends were ligated. The visceral branches of the pelvic nerves were transected bilaterally near the internal iliac vessels to prevent reflex bladder contractions. For the measurement of leak point pressure during sneezing (S-LPP), a PE-90 catheter connected to 
a pressure transducer (Transbridge 4M, World Precision Instruments, Sarasota, FL) was inserted into the bladder through the bladder dome for recording intravesical pressure. A handmade balloon catheter with a $1 \mathrm{~cm}$-diameter balloon connected to a pressure transducer was then inserted through a rectal incision into the abdominal cavity to record abdominal pressure (Pabd) during sneezing. The abdomen was then closed with sutures. Rats were then placed in a supine position for experimental testing.

\section{Measurements of urethral pressure responses}

The sneeze reflex was induced by inserting a rat's whisker gently into the nostril under urethane anesthesia as we previously reported $(4,5)$. After the bladder was emptied, a 3.5-Fr nylon catheter with a side-mounted microtransducer located $1 \mathrm{~mm}$ from the catheter tip (SPR-524, Millar Instruments, Houston, TX) was inserted into the middle portion of the urethra (10-15 mm from the urethral orifice), and urethral responses were recorded with data-acquisition software (sampling rate $400 \mathrm{~Hz}$; Chart, AD Instruments, Castle Hill, NSW, Australia) on a computer system equipped with an analog-to-digital converter (Power Lab, AD Instruments, Colorado Springs, CO). During experiment, rats were anesthetized by the intraperitoneal injection of urethane $(0.72 \mathrm{~g} / \mathrm{kg}$, Sigma Chemical Company, St. Louis, MO), and additional doses of anesthetic $(0.1 \mathrm{~g} / \mathrm{kg}$ per injection) were administered as required before starting sneeze reflex testing to obtain the sufficient level of anesthesia, which was confirmed by negative reflex responses to toe pinch. The final dose of urethane ranged 1.0 to $1.2 \mathrm{~g} / \mathrm{kg}$. The amplitude of urethral pressure responses during sneezing (A-URS) and urethral baseline pressure (UBP) were measured, as described in our previous reports $(4,5)$. 


\section{S-LPP (sneeze-induced leak point pressure)}

In a separate group of rats, the bladder was emptied and $0.4 \mathrm{ml}$ of saline solution containing Evans blue (100 mg/ml; Sigma, St. Louis, MO) was injected into the bladder through the intravesical catheter. The sneeze reflex was induced to examine whether fluid leakage from the urethral orifice was induced by sneezing. Intravesical pressure changes were recorded to monitor the increase in abdominal pressure during sneezing with the data acquisition software (sampling rate $400 \mathrm{~Hz}$; Chart, AD Instruments) on a computer system described above. The maximal intravesical pressure was measured in each sneeze event, and the lowest pressure value that induced fluid leakage from the urethral orifice was defined as S-LPP.

\section{Detection of apoptosis}

Cells containing fragmented DNA were visualized in urethral sections using the terminal deoxynucleotidyl transferase (TdT) mediated deoxyuridine triphosphate nick end-labeling method (TUNEL) using an ApopTag-In Situ Apoptosis Detection Kit (Chemicon, Temecula, CA, USA). In brief, frozen sections of the middle urethra were cut at $10 \mu \mathrm{m}$ thickness, washed twice with $50 \mathrm{mM}$ PBS, immersed in $0.5 \%$ Triton for $15 \mathrm{~min}$, washed twice, and digested with proteinase K (20 mug ml-1) for $15 \mathrm{~min}$, and endogenous peroxidase was quenched with $3 \% \mathrm{H}_{2} \mathrm{O}_{2}$ for 20 min. After two washings, the sections were incubated with TdT enzyme at $37{ }^{\circ} \mathrm{C}$ for $60 \mathrm{~min}$. This was followed by incubation with anti-digoxigenin-peroxidase for $60 \mathrm{~min}$ at room temperature, and color development with 
3\% $\mathrm{H}_{2} \mathrm{O}_{2}$ and 3, 3' diaminobenzidine tetrahydrochloride (Sigma, St. Louis, $\mathrm{MO}$ ) for $10 \mathrm{~min}$, followed by three washes in milli-Q water. Then, sections were counterstained with $0.5 \%$ methyl green and dehydrated in butanol followed by xylene and then covered with Permount mounting medium (Fisher, Fair Lawn, NJ). Specimens of post-weaning mammary gland tissue from rats were provided by Chemicon to use as positive control tissue. Omission of TdT enzyme from the incubation buffers during processing constituted the technical negative control. Apoptotic cells were observed in cross-section in randomly selected microscopic fields at a final magnification of $\times 400$.

\section{Apoptosis Index}

The percentage of TUNEL positive cells were calculated and used as the apoptotic index (AI). The number of TUNEL-positive cells in the designated area of the eight sections was summed, generating one value of total TUNEL-positive cells for each animal. The individual sums were averaged to obtain the group mean. The total number of TUNEL-positive cells in each animal was divided by the volume of the area examined to obtain the number of TUNEL-positive cells per $\mathrm{mm}^{3}$ of the urethral tissue for each animal. The individual numbers of TUNEL-positive cells per $\mathrm{mm}^{3}$ were then averaged to obtain the group means. All slides were read by a single researcher who was blinded to the experimental conditions. Sections were imaged on a color digital camera (Model 1KH027799; Olympus, Dulles, VA) on a BX45 Olympus microscope using MagnaFire software (version 2.1; Olympus). In the muscle layer of the middle urethra, outer striated muscle and inner smooth muscle layers were morphologically identified as the separate 
layers, as reported in previous studies (6).

\section{Statistical Analysis}

All data are expressed as means \pm SE. Excessively large sneezes that induced increases in Pabd greater than +2 SD above the average and very small responses inducing increases in Pabd $<3 \mathrm{cmH}_{2} \mathrm{O}$ were excluded from data analyses as we previously reported $(4,5)$. The values of the A-URS and UBP as well as increases in Pabd during sneezing were averaged in each rat. The mean \pm SE in a group of animals was then calculated from the averaged value in each rat of the same group. Repeated measures analysis of variance (ANOVA) followed by Tukey's multiple comparison test was used to compare changes in A-URS and UBP as well as increases in Pabd during sneezing. In the evaluation of apoptotic index, Student's t-test for unpaired data was used. All data were analyzed using the statistical software package Prism (Graphpad Software, San Diego, CA, USA). P values $<0.05$ were considered to be significant. 


\section{Results}

\section{Animal and uterus weight}

At 3 and 6 weeks after OVX in young rats, OVX rats had significantly higher body weights compared to time-matched young sham operated rats. Also, in middle-aged rats, the body weight of 6 weeks after OVX was significantly higher than in the intact group without OVX. In contrast, the weight of the uterus was significantly decreased at 3 and 6 weeks after OVX in both young (3 and 6 weeks) compared to intact and sham rats (Table 1), indicative of estrogen deficiency following OVX (7).

\section{Effects of OVX on urethral pressure responses in young and middle-aged rats}

In young rats, A-URS was significantly decreased in 3 and 6 weeks after OVX compared to time-matched, sham operated rats $\left(23.5 \pm 1.7\right.$ vs. $33.1 \pm 3.6 \mathrm{cmH}_{2} \mathrm{O}$ and $14.6 \pm 1.9$ vs. $31.4 \pm 2.8 \mathrm{cmH}_{2} \mathrm{O}$, respectively). UBP was significantly decreased only in 6-week OVX rats compared to sham rats $\left(14.6 \pm 0.9\right.$ vs. $\left.27.0 \pm 1.2 \mathrm{cmH}_{2} \mathrm{O}\right)$.

In intact middle-aged rats without OVX, UBP, but not A-URS, was significantly smaller than in intact young rats without OVX (UBP: $18.3 \pm 1.9$ vs. $26.7 \pm 1.7 \mathrm{cmH}_{2} \mathrm{O}$ ). In middle-aged rats with 3-week OVX, A-URS was significantly decreased compared to intact middle-aged rats or 3-week OVX young rats $(16.2 \pm 1.8$ vs. $29.5 \pm 3.9$ or $23.5 \pm 1.7$ $\mathrm{cmH}_{2} \mathrm{O}$, respectively, Table.2). In addition, the reduction in A-URS 3 weeks after OVX was significantly larger in middle-aged rats versus young rats (Table.2). 


\section{Effects of OVX on sneeze leak point pressure (S-LPP) in young and middle-aged rats}

Neither of intact rats (young or middle-aged), young rats with 3-week OVX or sham rats leaked during sneezing. However, SUI during sneeze occurred in 2 of 8 middle-aged rats with 3-week OVX. At 6 weeks of OVX, sneeze-induced SUI was observed in 5 out of 8 young rats and 6 out of 8 middle-aged rats with S-LPP values of 71.5 \pm 8.2 and $82.9 \pm 5.9$ $\mathrm{cmH}_{2} \mathrm{O}$, respectively (Table 3).

\section{TUNEL staining}

To examine whether aging and/or OVX causes apoptosis in the urethra, the TUNEL assay was performed. Microscopic examination showed that in the sham operated rat urethra, TUNEL-positive cells were hardly detected (Figure 2a). In contrast, in the urethra of middle-aged rats or those subjected to OVX, a number of TUNEL-positive apoptotic cells were observed. Positive staining was localized in the epithelium and sub-mucosal regions (Figure 2b, d), and in the area of striated muscle fibers (Figure 2c).

\section{Apoptosis index (AI)}

AI was significantly increased in urethral striated muscles, but not in the mucosa or smooth muscle, in middle-aged rats with or without OVX. OVX in young and old rats significantly increased AI in the mucosa (Figure 3). 


\section{Discussion}

Previous clinical studies have demonstrated that advancing age is associated with decreased urethral sphincter function in women (8) and that a decrease in the number of striated muscle cells in the human rhabdosphincter is found with an increase of apoptosis of the muscle fibers in the urethra of aged women $(9,10)$. However, the role of estrogen in urethral sphincter function remains controversial because it has been reported that hormone replacement therapy is not an effective treatment of postmenopausal SUI, with some reports showing even an adverse relationship (11). This report provides evidence that functional and morphological changes in the urethra may contribute to the high prevalence of SUI associated with aging and estrogen deficiency. The results indicate that: (1) aging is more likely to impair baseline urethral function (represented by UBP) than the sneeze evoked urethral striated muscle reflex contractions (represented by A-URS), although degeneration of striated muscle shown by increased AI values occurs in middle-aged rats, (2) OVX in young rats decreases A-URS even at an early stage (3 weeks postsurgery) and this effect increases further at 6 weeks when the animals exhibited SUI, (3) OVX induced significantly larger reductions in A-URS in middle-aged versus young animals and (4) OVX induces apoptotic changes (i.e., increased AI values) in the urethral mucosa of both young and middle-aged rats.

Although we did not check the serum estrogen concentrations, we observed increased body weight and decreased uterine weight after OVX compared with intact or sham operated rats in the present study (Table 1), indicating the success of surgical estrogen 
deprivation by OVX since changes in body and uterine weight are reportedly correlated with serum estrogen levels $(7,12)$. Moreover, Wang et al. (13) reported that the serum estradiol concentration of middle-aged (12 months old) female rats is not significantly different from young (3 months old) rats. Also, the uterine weight was similar between young and middle aged rats in this study (Table 1), suggesting that the serum estrogen level is not decreased in 12 months old rats.

We investigated the effect of aging and estrogen deficiency on the urethral closure mechanism during the sneeze reflex to determine if these two factors have synergistic effects. Neese et al. (14) studied 12 months old OVX rats as a middle-aged model. Also, Wang et al. utilized 12 months old and 18 months old rats as middle-aged and aged groups, respectively, in their study (13). Therefore, in this study, we used 12 months old rats as a middle-aged model. We previously examined the effect of estrogen deficiency on the urethral function in young rats (4) and showed that A-URS is decreased at an early stage (3 weeks after OVX) without inducing SUI during sneezing, and that further reductions of A-URS and UBP are associated with fluid leakage during sneezing at a later stage (6 weeks after OVX). This study further revealed that, prior to OVX, the UBP of middle-aged rats is lower than that of young rats, which is consistent with the results of clinical studies in post-menopausal women (15). The effect of OVX-induced estrogen deficiency then further damages urethral function, leading to overt SUI in middle-aged rats with 3-week OVX, which was not seen in young rats with 3-week OVX. Taken together, these results suggest that aging and estrogen deficiency can additively impair the urethral continence mechanism to induce SUI under stress conditions such as sneezing. 
In functional testing in the present study, middle-aged rats (12 months old) showed a reduction in UBP without affecting A-URS; whereas OVX progressively reduced both A-URS and UBP. We previously reported that increased urethral pressure during sneezing (i.e., A-URS) is mediated by striated muscle contraction of the external urethral sphincter and the pelvic floor due to activation of the pudendal nerves and the somatic nerves innervating the pelvic floor muscles, respectively (3). Thus, the decrease in A-URS after OVX in this study indicates impaired striated muscle-mediated continence mechanisms. In contrast, UBP seems to reflect urethral smooth muscle activity since in our pervious study the increase in UBP was blocked by $\alpha$-adrenoceptor antagonists such as prazosin or by hypogastric nerve transaction $(3,16)$. Thus, UBP is likely to represent the baseline, coapting urethral function including urethral smooth muscle activity. Thus, our results suggest that OVX and the aging process affect the baseline urethral coapting function as evidenced by the reduced UBP values and that OVX additionally impairs striated muscle mediated urethral continence mechanisms (i.e., A-URS reduction) in middle-aged rats. These results seem to be in line with the findings in previous studies that aging and estrogen deficiency induce functional and morphological changes in the urethra, resulting in SUI in a rat model (17) and that the urethral pressure profile measurement revealed a lower maximum urethral closure pressure in long term (9 months) OVX rats compared to control animals (18).

Apoptosis is the most common form of physiological cell death and plays an important role in normal development and homeostasis of various tissues (19), and has also been linked to a wide variety of pathophysiological conditions (2). TdT-mediated 
dUTP-biotin nick end-labeling (TUNEL) methods are widely used for the detection of apoptotic cells. In the current study, apoptosis was examined to explore the changes in the urethral structures during the aging and estrogen deficiency process. Our results showed that the number of TUNEL-positive apoptotic cells in the striated muscle was increased in middle-aged rats. These results are consistent with previous clinical observations in cadavers of elderly people showing that an age-dependent increase of apoptosis of the striated muscle fibers is associated with a decrease in the number of striated muscle cells in the human rhabdosphincter $(9,10)$. However, in this study, the striated muscle activity of the urethral sphincter in response to sneezing (ie., the A-URS) was not significantly changed in middle-aged rats (12 months old) despite the increased AI values of the urethral striated muscle. Thus it is likely that, in rats at the age of 12 months, even though apoptotic changes have already started in the urethral striated muscle sphincter; the neurally evoked continence reflex mediated by striated muscle contraction is still functioning. Further studies using rats older than 12 months may be needed to clarify the functional aspect of apoptosis-related changes in the urethral striated muscle sphincter during the aging process.

The present study also found that the number of apoptotic cells significantly increased in the mucosa after OVX, which is consistent with the results in a previous study by Resplande et al. showing a similar increase in the number of apoptotic cells in the rat urethral mucosa 9 months after OVX (18). Estrogen receptors are widely distributed in the urogenital organs $(20,21)$ and, in female rats, apoptosis is observed not only in the urethra, but also in the vagina and bladder after $\operatorname{OVX}(20,21)$. Thus, these apoptotic changes in the urethral mucosa due to estrogen deficiency might contribute to the 
impairment of mucosal coaptation and/or the decrease of vascularity and tone of the urethra, which could reduce the baseline urethral resistance (i.e., decreased UBP) (4).

However, this study also showed that UBP was reduced in middle-aged rats without inducing apoptotic changes in the urethral mucosa. We have recently reported preliminary results showing that the reduction of UBP in middle-aged rats is associated with the remodeling of the urethral extracellular matrix (ECM) including reductions in collagen (type 1 and 3) and lysyl oxidase (22). These results suggest that the reduction in baseline urethral resistance (i.e., decreased UBP) in middle-aged rats is due to ECM remodeling, which may attenuates stiffness and elasticity of the urethral supporting system, although further studies are needed to clarify this point.

In addition to direct effects on the urethral target organs, OVX can also influence the neural control of the urethra. This has been detected as a change in the bursting pattern of the external sphincter electromyogram (EUS EMG) as well as a change in voiding function (23). In rats 4-12 weeks after OVX, the silent period of the EUS EMG and voiding efficiency are significantly reduced, and postvoid residual volume is increased. These changes that were prevented by estradiol treatment are most likely related to an alteration in hormone sensitive central neural mechanisms that coordinate bladder and sphincter activity. Similar neural changes could contribute to the reduction in sneeze evoked A-URS after OVX.

There are some limitations of our study. First, we used 12 months old rats to examine the effects of aging process on urethral function. However, they are still in their middle age; therefore, future studies are needed to study rats older than 12 months to further clarify 
the age-related pathophysiology of SUI. Secondly, OVX rats were used to induce estrogen deficiency because Wang et al. (13) reported that the serum estradiol concentration of middle-aged (12 months old) female rats was not significantly different from that of young (3 months old) rats. However, the complete lack of estrogen induced by OVX may not represent the situation in peri- to post-menopausal women, whose estrogen levels are gradually decreased. Lastly, although we focused on apoptotic changes associated with aging and estrogen deficiency in this study, other mechanisms such as nerve or muscle dysfunction and changes in extracellular matrix proteins (e.g., collagen or elastin) could contribute to urethral dysfunction after aging and estrogen deficiency. Further studies are necessary to elucidate other potential mechanisms than apoptosis that underlie the age or estrogen deficiency-related urethral dysfunction leading to SUI.

\section{Conclusion}

Our results indicate that the aging process and estrogen deficiency differently affect the baseline urethral function and the neutrally-evoked, striated muscle-mediated urethral continence mechanism to induce SUI under stress conditions such as sneezing. Thus, these two etiological factors may additively contribute to the emergence of SUI in post-menopausal women. 


\section{Acknowledgements}

This study was supported by National Institutes of Health grants (NIH DK067226, AR049398 and DK055387). 


\section{REFERENCES}

1. Brown JS, Grady D, Ouslander JG, et al. Prevalence of urinary incontinence and associated risk factors in postmenopausal women. Heart \& Estrogen/Progestin Replacement Study (HERS) Research Group. Obstet Gynecol. 1999;94:66-70.

2. Kerr JF, Wyllie AH, Currie AR. Apoptosis: a basic biological phenomenon with wide-ranging implications in tissue kinetics. Br J Cancer. 1972;26:239-57.

3. Kamo I, Torimoto K, Chancellor MB, et al. Urethral closure mechanisms under sneeze-induced stress condition in rats: a new animal model for evaluation of stress urinary incontinence. Am J Physiol Regul Integr Comp Physiol. 2003;285:R356-65.

4. Kitta T, Haworth-Ward DJ, Miyazato M, et al. Effects of ovariectomy and estrogen replacement on the urethral continence reflex during sneezing in rats. J Urol. 2011;186:1517-23.

5. Kitta T, Miyazato M, Chancellor MB, et al. Alpha2-adrenoceptor blockade potentiates the effect of duloxetine on sneeze induced urethral continence reflex in rats. J Urol. 2010;184:762-8.

6. Lim SH, Wang TJ, Tseng GF, et al. The distribution of muscles fibers and their types in the female rat urethra: cytoarchitecture and three-dimensional reconstruction. Anat Rec (Hoboken). 2013;296:1640-9.

7. Yoshida J, Aikawa K, Yoshimura Y, et al. The effects of ovariectomy and estrogen replacement on acetylcholine release from nerve fibres and passive stretch-induced acetylcholine release in female rat bladder. Neurourol Urodyn. 2007;26:1050-5. 
8. Kenton K, Mueller E, Brubaker L. Neuromuscular characterization of the urethra in continent women. Female Pelvic Med Reconstr Surg. 2011;17:226-30.

9. Strasser H, Tiefenthaler M, Steinlechner M, et al. Age dependent apoptosis and loss of rhabdosphincter cells. J Urol. 2000;164:1781-5.

10. Strasser H, Tiefenthaler M, Steinlechner M, et al. Urinary incontinence in the elderly and age-dependent apoptosis of rhabdosphincter cells. Lancet. 1999;354:918-9.

11. Hendrix SL, Cochrane BB, Nygaard IE, et al. Effects of estrogen with and without progestin on urinary incontinence. JAMA. 2005;293:935-48.

12. Guo HY, Jiang L, Ibrahim SA, et al. Orally administered lactoferrin preserves bone mass and microarchitecture in ovariectomized rats. J Nutr. 2009;139:958-64.

13. Wang VC, Neese SL, Korol DL, et al. Chronic estradiol replacement impairs performance on an operant delayed spatial alternation task in young, middle-aged, and old rats. Horm Behav. 2009;56:382-90.

14. Neese SL, Korol DL, Katzenellenbogen JA, et al. Impact of estrogen receptor alpha and beta agonists on delayed alternation in middle-aged rats. Horm Behav. 2010;58:878-90.

15. Sorensen S. Urodynamic investigations and their reproducibility in healthy postmenopausal females. Scand J Urol Nephrol Suppl. 1988;114:42-7.

16. Kaiho Y, Kamo I, Chancellor MB, et al. Role of noradrenergic pathways in sneeze-induced urethral continence reflex in rats. Am J Physiol Renal Physiol. 2007;292:F639-46.

17. Russell B, Baumann M, Heidkamp MC, et al. Morphometry of the aging female 
rat urethra. Int Urogynecol J Pelvic Floor Dysfunct. 1996;7:30-6.

18. Resplande J, Gholami SS, Graziottin TM, et al. Long-term effect of ovariectomy and simulated birth trauma on the lower urinary tract of female rats. J Urol. 2002;168:323-30.

19. Raff MC, Barres BA, Burne JF, et al. Programmed cell death and the control of cell survival: lessons from the nervous system. Science. 1993;262:695-700.

20. Aikawa K, Sugino T, Matsumoto S, et al. The effect of ovariectomy and estradiol on rabbit bladder smooth muscle contraction and morphology. J Urol. 2003;170:634-7.

21. Wolf $\mathrm{H}$, Wandt $\mathrm{H}$, Jonat W. Immunohistochemical evidence of estrogen and progesterone receptors in the female lower urinary tract and comparison with the vagina. Gynecol Obstet Invest. 1991;32:227-31.

22. Sumino $\mathrm{Y}$, Yoshikawa S, Mimata $\mathrm{H}$, et al. Association of remodelling of extracellular matrix and urethral dysfunction inducing stress urinary incontinence in aged, multiparous rats. Neurourol Urodyn. 2013;32:831-2.

23. Cheng CL, de Groat WC. Effect of ovariectomy on external urethral sphincter activity in anesthetized female rats. J Urol. 2011;186:334-40. 
Figure legends

Fig.1 Schema showing the experimental schedule of different rat groups. 
Fig.2 TUNEL (terminal deoxynucleotidyl transferase mediated deoxyuridine triphosphate nick end-labeling method) staining of urethral tissues from sham operated young (a), ovariectomized young (b), intact middle-aged (c) and ovariectomized middle-aged rats (d). Positive brown color staining indicated by arrowheads was localized in the epithelium and submucosal regions (b, d), and in striated muscle fibers (c). The sham operated young rat shows only a few apoptotic cells (a). Reduced from $\times 400$. L represents the urethral lumen. 
Fig.3 Apoptotic index indicating the percent of TUNEL positive cells/total number of cells counted. The results are expressed as mean \pm SE ( $n=5-7$ per group).

Total: apoptotic index in the entire urethral section, MU: apoptotic index in the mucosal layer of urethral sections, SM: apoptotic index in the smooth muscle layer of urethral sections, ST: apoptotic index in the striated muscle layer of urethral sections ${ }^{*} \mathrm{p}<0.05$ versus Sham or Intact rat groups, ${ }^{* *} \mathrm{p}<0.01$ versus Sham or Intact rat groups, $† \mathrm{p}$ $<0.05$ versus young rat groups. 
Fig.1

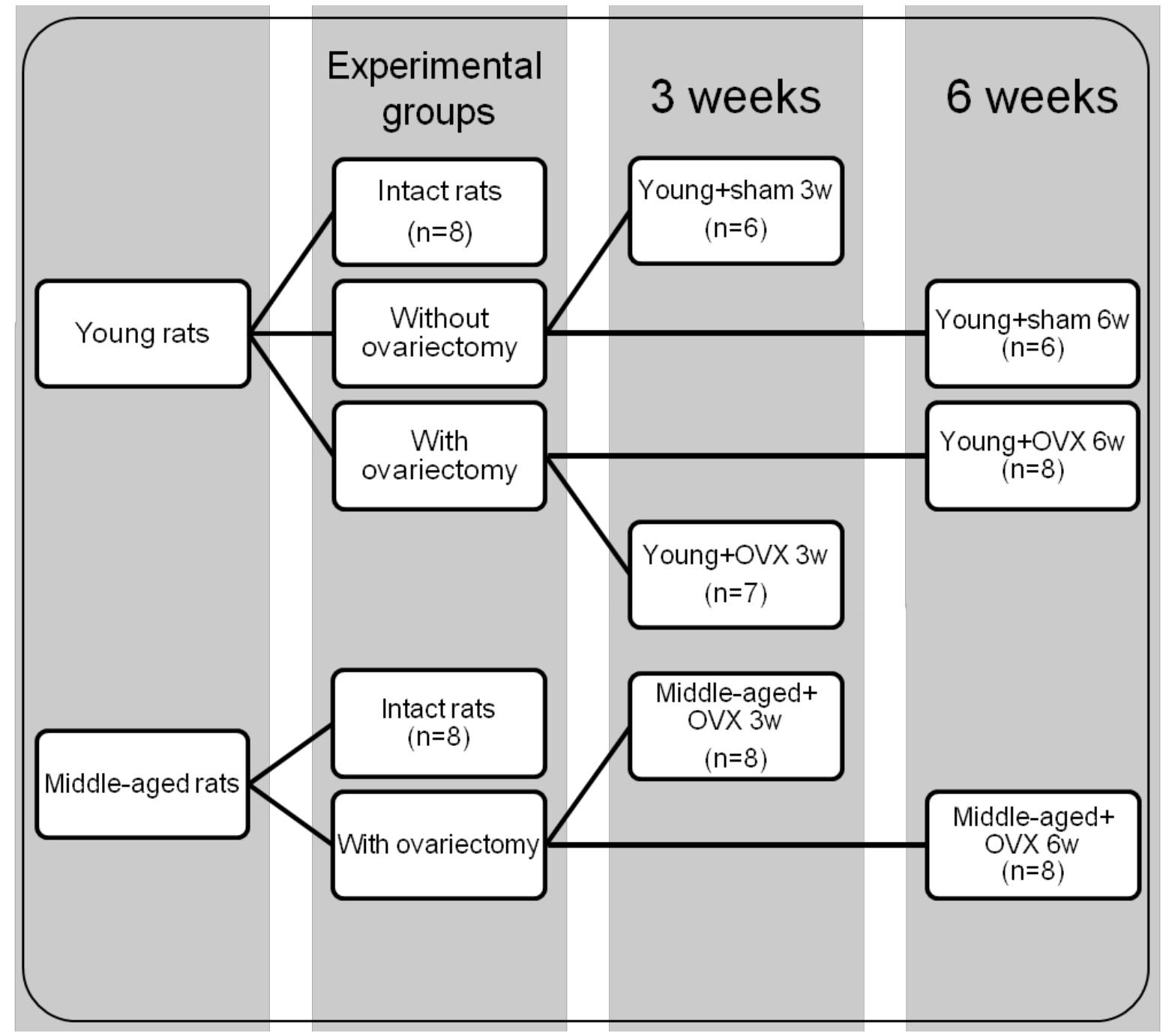


Fig. 3

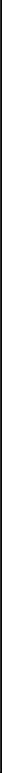




\section{Table 1}

Animal and tissue weights in young and middle-aged rats

\begin{tabular}{|c|c|c|c|c|c|c|c|c|}
\hline \multirow[b]{3}{*}{ Rat weight (g) } & \multicolumn{5}{|c|}{ Young rats } & \multicolumn{3}{|c|}{ Middle-aged rats } \\
\hline & Intact & Sham 3w & Sham 6w & OVX 3w & OVX6w & Intact & OVX 3w & OVX6w \\
\hline & $262 \pm 4$ & $269 \pm 4$ & $286 \pm 6$ & $294 \pm 6^{*}$ & $348 \pm 8^{*}$ & $355 \pm 8$ & $366 \pm 12$ & $379 \pm 7^{\dagger}$ \\
\hline Uterus wet weight (g) & $0.81 \pm 0.05$ & $0.82 \pm 0.06$ & $0.78 \pm 0.05$ & $0.42 \pm 0.04^{\star}$ & $0.36 \pm 0.04^{\star}$ & $0.84 \pm 0.03$ & $0.43 \pm 0.02^{\dagger}$ & $0.42 \pm 0.04^{\dagger}$ \\
\hline
\end{tabular}

Values $(\mathrm{g})$ are means $\pm \mathrm{SE} .{ }^{*} \mathrm{P}<0.05$ versus time-matched sham operated rats, ${ }^{\dagger} \mathrm{P}<0.05$ versus intact aged rats. $(n=8-12)$ Sham; sham operated rats, OVX; ovariectomized rats 


\section{Table 2}

\section{Urethral functions of different groups of rats}

\begin{tabular}{|c|c|c|c|}
\hline \multicolumn{4}{|c|}{ A-URS } \\
\hline & \multicolumn{2}{|c|}{ Young } & Middle-aged \\
\hline Intact & \multicolumn{2}{|c|}{$29.0 \pm 3.3$} & $29.5 \pm 3.9$ \\
\hline & Sham & oVX & ovX \\
\hline 3weeks & $33.1 \pm 3.6$ & $23.5 \pm 1.7^{\star}$ & $16.2 \pm 1.8 \dagger \#$ \\
\hline 6weeks & $31.4 \pm 2.8$ & $14.6 \pm 1.9^{*}$ & $14.0 \pm 2.6 \dagger$ \\
\hline \multicolumn{4}{|c|}{ UBP } \\
\hline & \multicolumn{2}{|c|}{ Young } & Middle-aged \\
\hline Intact & \multicolumn{2}{|c|}{$26.7 \pm 1.7$} & $18.3 \pm 1.9 \dagger$ \\
\hline & Sham & oVX & ovX \\
\hline 3weeks & $27.0 \pm 0.8$ & $21.7 \pm 2.0$ & $19.6 \pm 1.0 \dagger$ \\
\hline 6weeks & $27.0 \pm 1.2$ & $14.6 \pm 0.9^{*}$ & $15.7 \pm 1.4 \dagger$ \\
\hline
\end{tabular}

All values are expressed in $\mathrm{cmH}_{2} \mathrm{O}$.

${ }^{*} P<0.05{ }^{* *} P<0.01$ compared with control values of intact rats (Intact) in the respective group.

$\dagger P<0.05$ compared with the same timeline sham group.

$\# P<0.05$ compared with the same timeline OVX group. 


\section{Table 3}

The number of SUI rats and S-LPP (mean $\pm \mathrm{SE} \mathrm{cmH}_{2} \mathrm{O}$ in parentheses) of different groups of rats

\begin{tabular}{|l|l|l|l|l|}
\hline & Intact & \multicolumn{2}{|c|}{ 3 weeks } & 6 weeks \\
\hline \multirow{2}{*}{ Young } & \multirow{2}{*}{ /8 } & Sham & $0 / 6$ & $0 / 6$ \\
\cline { 3 - 5 } & & OVX & $0 / 7$ & $5 / 8\left(71.5 \pm 8.2 \mathrm{cmH}_{2} \mathrm{O}\right)$ \\
\hline Middle-aged & $0 / 8$ & OVX & $2 / 8\left(76.9 \pm 10.2 \mathrm{cmH}_{2} \mathrm{O}\right)$ & $6 / 8\left(82.9 \pm 5.9 \mathrm{cmH}_{2} \mathrm{O}\right)$ \\
\hline
\end{tabular}

Values are means $\pm \mathrm{SE}$.

Sham; sham operated rats, OVX; ovariectomized rats 